\title{
A Review on the Use of Rapid Small Scale Column Test (RSSCT) on Predicting Adsorption of Various Contaminants
}

\author{
Moumita Poddar ${ }^{1 *}$, A.N. Brijesh Nair ${ }^{2}$, Amit B Mahindrakar ${ }^{2}$ \\ ${ }^{1}$ Postgraduate Student, ${ }^{2}$ Professor, School of Mechanical and Building Sciences, VIT University, Vellore - 632014, \\ TamilNadu, India
}

\begin{abstract}
The Rapid Small Scale Column Test (RSSCT) is a small-scale model of a pilot-scale system which is aimed to give performance equal to the full-scale system when loaded with small sized particle adsorbent such as Granular Activated Carbon (GAC). The RSSCT was developed to predict the exclusion of organic compounds on activated carbon adsorbers. The theory behind RSSCT is to scale down the hydrodynamic characteristics and mass transfer phenomena from a pilot scale reactor to a small scale flow test column as it is expected that the breakthrough curves would be similar for both of them. As compared to the pilot scale columns, the RSSCT requires only a portion of water and time to stimulate a several month's pilot scale study into a few days adsorption study under laboratory conditions. The RSSCT is used widely to predict the performance for various adsorption systems as they are inexpensive and gives a constant flow test results within few days which means that RSSCT are more representative as compared to the batch tests. Apart from these advantages RSSCT can be used to compare the efficiency of media and the quality of water. Usually the adsorbent used in RSSCT study is GAC which is first activated using different processes to increase the adsorptive capacity and surface reactivity and then it is grounded to a specific size fraction which can be used for RSSCT study. Sometimes grinding of adsorbents may be required if the sorption properties varies. This review study would give a detailed insight in designing a RSSCT model on a laboratory scale, scaling adsorption capacity of trace organic contaminant by various adsorbents and to predict a full scale breakthrough.
\end{abstract}

Keywords: RSSCT, GAC, Breakthrough curves, Adsorption, Adsorbents

\section{Introduction}

The rapid small-scale column test (RSSCT) is a quick method to design a large-scale adsorbent column from small scale column studies. The RSSCT can be used to successfully replicate a pilot scale test column study for empty bed contact time (EBCT). In previous research works, a scaling relationship between hydraulic loadings and EBCTs of the pilot scale test column and RSSCT was established for various particle adsorbent sizes. Apart from these, different scaling parameters are needed to match RSSCT breakthrough profiles with the corresponding pilot-scale adsorbent studies like intraparticle diffusion along with a controlled adsorption rate. The only thing required in designing the pilot scale column are, understanding the breakthrough and column tests which is required for conducting the experiments.

\subsection{Background}

In pilot scale columns it takes months to achieve a breakthrough curve but with the RSSCT model the breakthrough can be achieved in hours or days which represent the data of pilot scale test results. For designing the RSSCT columns, it is necessary to ground the GAC to small particle size of specific range according to the requirement. The use of RSSCT for a design has many advantages such as: There is no requirement of kinetic studies to predict the performance of a full scale column from RSSCT, It can be easily carried out under controlled laboratory conditions within few days, only a small amount of water is required for testing and the procedures are relatively inexpensive [1].

\subsubsection{Mass Transport in Porous Adsorbents}

Porous adsorbents have high surface area and the mass transport of ions from the solution on to the adsorbent takes place by film diffusion or pore diffusion. Apart from diffusion technique there is also intraparticle transport which is a combination of these diffusion processes. The adsorption of pollutant concentration is highest on external surface and minimum in the interior, which creates a concentration gradient and allows adsorption on the porous media; therefore we can say that intraparticle transport is rate limiting mechanism [2]. But with time the adsorption of the pollutant slows down and thus we can measure increased pollutant concentration in the effluent.

In order to get a successful breakthrough profile in RSSCT the following assumptions are needed: backwashing need not be taken into consideration as it may obliterate the mass transport zone, the scaling procedure should be based on dispersed-flow, pore-surface-diffusion model (DFPSDM), it is not possible to 
regenerate GAC in a short duration of time through biological activity, the limiting factor is internal mass transfer which means that the media should be micro porous in nature, Distribution of pore size should be independent of the radius of the particle or the mesh size, there should be no variations in the properties of GAC such as the adsorption capacity, porosity and bulk density, when it is crushed from complete-size to minute-size, the interaction of the target compound with the adsorption sites should depend on the size of the particle [2]. The various parameters which are assumed for large columns are: EBCT, Bed Length, Hydraulic loading rate, Mean particle size, internal diameter of the column, depth of media and flow rate [3].

\subsubsection{Approaches in Designing RSSCT Model}

There are two approaches to design RSSCT model; the constant diffusivity (CD-RSSCT) test which assumes that there is no reliance of intraparticle diffusivity on the particle size of GAC, and the proportional diffusivity (PD-RSSCT) test which assumes that there exists a linear proportionality between intraparticle diffusivity on the particle size of GAC, which shows a superior outcome when predicting the breakthrough of DOM as measured by dissolved organic carbon (DOC). But on the other hand showed the CD-RSSCT could predict the breakthrough curves of organic contaminants in the absence of DOM. It has been found by extensive research that the PD-RSSCT should be used in the removal of DOM, which causes fouling [4]. It is found that the high molecular weight compounds are prone to size exclusion in small GAC pores, which ultimately leads deliberate adsorption of a target compound which is because of the hysterical site competition by the competing compounds which have the same molecular weight as the target compound [5].

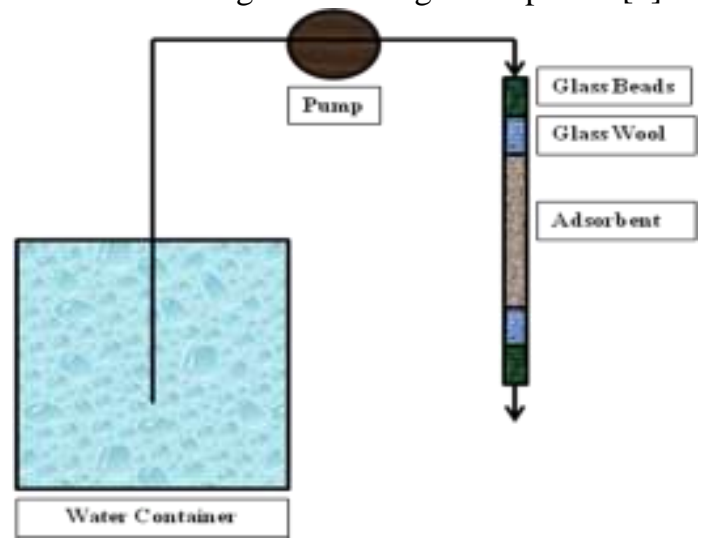

Fig.1. Schematic of the Rapid Small-Scale Column Test Apparatus

\subsubsection{Similitude in Operation of RSSCT and Pilot Scale Column}

Similarity in operation of RSSCT and pilot scale column is achieved by proper selection of hydraulic loading rate, EBCT (Empty Bed Contact Time) and particle size (Fig.1). The two columns should be similar with respect to surface diffusivities, bulk density, temperature and concentration, in order to achieve similar breakthrough profiles [1]. The equation for calculating the same is represented as follows in equation (1)

$$
\frac{\mathrm{EBCT}_{S C}}{\mathrm{EBCT}_{\mathrm{LC}}}=\frac{\left[\mathrm{d}_{\mathrm{p}, S C}\right]^{2-x}}{\left[\mathrm{~d}_{\mathrm{p}, \mathrm{LC}}\right]^{2-x}}=\frac{\mathrm{t}_{S C}}{\mathrm{t}_{\mathrm{LC}}}
$$

$\mathrm{X}=$ depends on pore diffusivity ( 0 or 1 for $\mathrm{CD}$ and $\mathrm{PD}$ respectively)

$\mathrm{d}_{\mathrm{p}}=$ Particle diameter

$\mathrm{t}=$ time

$\mathrm{SC}=$ Small column

$\mathrm{LC}=$ Long column

$\mathrm{V}_{\mathrm{i}}=$ Hydraulic loading rate

$\mathrm{V}_{\text {bed }}=$ Volume of bed

$\mathrm{Q}=$ Flow Rate

The hydraulic loading rate is scaled based on the relation given as follows in equation (2):

$$
\frac{V_{i, L C}}{V_{i, S C}}=\frac{d_{p, L C}}{d_{p, S C}}
$$

The EBCT is scaled based on Volume of bed and Flow Rate given as follows in equations (3) and (4): 


$$
\begin{aligned}
\mathrm{EBCT} & =\frac{\mathrm{V}_{\text {bed }}}{\mathrm{Q}} \\
\text { Bed volume treated } & =\frac{\left(\mathrm{Q}^{*} \mathrm{~T}\right)}{\mathrm{V}_{\text {bed }}}=\frac{\mathrm{T}}{\mathrm{EBCT}}
\end{aligned}
$$

\subsubsection{Rapid Small Scale Column Test using Porous Adsorbents}

The adsorbent which is widely used in treating water or waste water for the removal of pollutants such as Total Organic Compounds (TOCs), Natural Organic Compounds (NOCs) and Synthetic Organic Compounds (SOCs) is Granular Activated Carbon (GAC). This method of treating water is extensively used because it is relatively inexpensive.

The adsorptive properties of GAC can be varied by demineralising it with Hydrogen fluoride (HF) and Hydrogen chloride $(\mathrm{HCl})$ and then oxidizing it with $\mathrm{HNO}_{3}[6]$. Thus it is important to take into consideration that it is the distribution of pore and the physical characteristics of the surface determines the adsorptive nature of the activated carbon [6]. The various preparatory materials for the manufacture of GACs are wood char, bituminous coal, and lignite coal [7]. The adsorption pattern of GAC is different at the surface and in the pores [7].

It is sometimes necessary to grind adsorbent particles if there is some variation in adsorption pattern. Sometimes due to the variation in the physical characteristics of surface properties, the RSSCT results can be subjective because the entire RSSCT method is dependent on the specific size ranges of smaller GAC particles having adsorption properties analogous to that of the bulk adsorbents [8]. This would eventually nullify the technique, but RSSCT method can be considered valid only when there would be no considerable change in the surface characteristics of bulk adsorbents and its grounded fractions [9]. In case of dissolved organic matter (DOM), RSSCT results have not been able to successfully stimulate the pilot scale data. Hence the effectiveness of RSSCT is largely dependent on the type of adsorbents used in the media and the optimum EBCT required to scale down the results [9].

\subsection{Scope of Study and objectives}

This review will give a detailed knowledge on adsorption of various pollutants using GAC, determining the breakthrough curve for specific pollutants, with proper understanding of the concept of RSSCT and predicting the pilot scale adsorption phenomena.

The aim of this review is to introduce to the concept of RSSCT and to determine the use of RSSCT to predict the pilot scale behaviour of adsorbents in recent years. Specifically the objectives of this review are:

\subsubsection{Introduction to the concept of RSSCT}

- Feasibility of using adsorbents in removing contaminants from drinking water using RSSCT.

- Mass Transport in Porous Adsorbents using Rapid Small Scale Column Test

- Approaches in Designing RSSCT Model Similitude in Operation with Pilot Scale Column

1.2.2 Use of RSSCT to predict the pilot scale behaviour of adsorbents

- Effectiveness of RSSCT with different particle diameter.

- Cost evaluation of synthetic resin technology.

- Applications of RSSCT in contaminant removal.

\section{Literature Review}

This section reviews the literature relevant to the objectives of the present study. Detailed literature on the following aspects have been collected and given here under the following sections.

\subsection{Arsenic Removal using RSSCT}

In recent times the maximum contaminant level (MCL) of arsenic in drinking water has been lowered by Environmental Protection Agency (EPA) to $10 \mathrm{ppb}$ from $50 \mathrm{ppb}$, as arsenic has detrimental effect if consumed [10]. RSSCT has been used to evaluate the capacity of diverse adsorptive media to remove oxyanions of uranium, antimony, phosphorous, arsenic and vanadium. It has been observed that the RSSCT has the capacity to remove a concentration of $7 \mathrm{ppb}$ arsenic in source water from the initial concentration of $13 \mathrm{ppb}$ [10]. Oxygenated waters contain arsenic in anionic form in the $\mathrm{pH}$ range of 5 to 12 [10]. Usually the dominant form of arsenic is Arsenate $\left(\mathrm{H}_{3} \mathrm{AsO}_{4}, \mathrm{H}_{2} \mathrm{AsO}_{4}{ }_{4}^{-}, \mathrm{HAsO}_{4}{ }^{2-}\right.$, or $\mathrm{AsO}_{4}{ }^{3}$ or it may be also present in non ionic form $\left(\mathrm{H}_{3} \mathrm{AsO}_{3}\right)$ below $\mathrm{pH}$ 9.2. Arsenite is readily oxygenated to arsenate by the oxidation of Chlorine, permanganate, or ozone. Arsenic has been effectively removed using metal oxides as adsorbent by electrostatic force of attraction and covalent bonding on the positively charged surface but in case of GAC as adsorbent being used, then there exists hydrophobic bonding [11]. The removal of arsenic can be indicated by the $\mathrm{pH}$ zero point of charge $\left(\mathrm{pH}_{\mathrm{ZPC}}\right)$. The major anion that effects the removal of arsenic is silica as it reduces the adsorptive 
capacities of ferric oxides/hydroxides and activated alumina. This happens because the silica used decreases the iso-electric point, competes with arsenic absorption sites and chemically reacts [11]. The important factors that affect the performance of adsorption media are selectivity, capacity and adsorption kinetics. Geochemically the media can be characterised by electron microscopy, surface area analysis studies and by studying the $\mathrm{pH}_{\mathrm{ZPC}}$. Thus, RSSCT technique has been developed to remove arsenic from source water, which is a dimensionless mathematical formula. The first thing that is kept in mind is that boundary conditions should occur at the same dimensionless coordinate and secondly there should be no change in mass transfer on reducing the size of the column [10].

A stand-alone RSSCT apparatus was designed for the successful removal of arsenic, which was 30.5 $\mathrm{cm}$ in length with Teflon end capping, $1.2 \mathrm{~cm}$ in diameter, borosilicate glass beads and glass wool were used for packing the column making sure that there is no air bubbles [5]. Crushed and sieved homogeneous media was used in the column based on the parameter decided in designing the column. Then reverse grading was done to compress the media and to obtain a required bed depth until the effluent was visibly clear. The calibrated flow rate of the pump was checked by passing distilled water for 15 minutes. Experiments were conducted to test the arsenic levels in source groundwater [11].

The effluent collected from RSSCT was then tested through analytical procedure to estimate the desirable and permissible limit of arsenic in water. TOC tests were performed to determine the level of toxicity in groundwater and Varian ${ }^{\circledR}$ graphite furnace atomic absorption spectrophotometer was also used for the analysis. The comparison of small and pilot scale studies showed the same media performance with smoother breakthrough curves in laboratory studies with steady $\mathrm{pH}$, temperature and concentration. These experiments showed the best arsenic removal treated to $10 \mu \mathrm{g} / \mathrm{L}$ breakthrough [10].

\subsubsection{Metal Oxyhydroxides Adsorbents for Removal of Arsenic}

Oxygen atoms are found on the surface of metal oxides, which gets attached to the hydrogen atoms from the neighbouring water which acts as ligands [12]. This interaction greatly depends on the pH. Metal hydroxides are porous adsorbents which results in interfacial interaction between adsorbates and adsorbents and have been successfully applied in RSSCT.

\subsubsection{Activated Alumina Adsorbents for Removal of Arsenic}

Alumina is activated by dehydrating aluminium hydroxide at 300-600 $\mathrm{C}$ [10]. Activated alumina is amorphous, crystalline, porous and inorganic. It has been successfully used in the removal of arsenic from source water. The smaller the particle size, the larger is the surface area and increased adsorption of arsenic. The surface charge of activated alumina is dependent on the $\mathrm{pH}$. The $\mathrm{pH}_{\mathrm{ZPC}}$ activated alumina is around 8.2, which means that there will be positive surface charge and electrostatic force of attraction if the $\mathrm{pH}$ is less than 8.2 , but if the $\mathrm{pH}$ is greater than 8.2 than the charge on surface will be negative will adsorb cations [10]. Thus arsenate can be easily adsorbed. Care has to be taken as activated alumina dissolves at very low $\mathrm{pH}$ [12].

\subsubsection{Ferric Hydroxide Adsorbents for Removal of Arsenic}

Ferric hydroxide is crystalline in nature with a porosity of 70-80\% [12]. The removal arsenic is also strongly dependent on $\mathrm{pH}$ like the other adsorbents [12]. Increased surface area, high $\mathrm{pH}_{\mathrm{ZPC}}$ is required for the formation of covalent bonds and the $\mathrm{pH}$ during treating the source water should be less than $\mathrm{pH}_{\mathrm{ZPC}}$ so as to provide high electrostatic force of attraction.

\subsection{Removal of Bromated Ion by GAC using RSSCT}

Ozonated water containing bromide ions results in the formation of bromated ions and brominated organics. These ions are strong oxidants and care carcinogenic in nature which causes malignant tumours in human beings if consumed. The MCL for bromated ion as formulated by EPA in drinking water should be less than $10 \mu \mathrm{g} / \mathrm{L}$ [3]. The disinfection process using ozone process produces chlorinated THMs and haloacetic acids (HAAs) in the presence of natural organic matter (NOM) [15]. Various strategies have been formulated to remove bromated ions by decreasing $\mathrm{pH}$, by addition of ammonia and hydrogen peroxide, but still there exists the formation of organic compounds in the presence of NOM, which are not feasible and cost effective [13].

It has been found that the bromated ion formed during ozonation can be successfully removed by treating with ultraviolet (UV) irradiation whose efficiency greatly depends on pressure and wavelength used or by using GAC and by using ultra filtration and nanofiltration [14]. But all these techniques except GAC are not cost effective due to high expenditure in operating and capital expenses [23]. GAC can be used in RSSCT to remove bromated ions using chemical reduction techniques. It has higher efficiency at lower $\mathrm{pH}$. GAC can be used in removal of organic carbon and chlorates with reduced time and efforts as compared to pilot scale studies under same conditions [7]. 
The experiments were conducted with the initial influent concentration of $50 \mu \mathrm{g} / \mathrm{L}$ of bromated ion, $\mathrm{pH}$ of 6, which was later adjusted to 8 according to the experimental requirements. $\mathrm{NaBrO}_{3}$ solution was spiked in water tank having $50 \mathrm{mg} / \mathrm{L}$ of $\mathrm{BrO}_{3}{ }^{-}$ion concentration [14]. Using the methodology developed by Crittenden, et $a l$, RSSCT experiments were conducted based on dynamic flow to obtain breakthrough curve. The pilot-scale experiments were stimulated with 10-min EBCT which resulted in $96 \%$ removal of $\mathrm{BrO}_{3}{ }^{-}$ion [14]. The media GAC was grinded and sieved to achieve desired particle size and was washed with organic free water to remove fine particles.

Ions such as $\mathrm{BrO}_{3}{ }^{-}$and $\mathrm{Br}^{-}$were analyzed using ion chromatography with borate as eluent and the samples were passed through columns which were metal free. DOC was analysed in TOC analyzer based on the method of sodium persulfate oxidation and suspended particles were removed in a filter paper of $0.45 \mu \mathrm{m}$ [14]. For $\mathrm{BrO}_{3}{ }^{-}$ions the breakthrough curves were steeper as the effluent approached the influent concentration. But $\mathrm{Br}^{-}$ions got completely removed in the steep curve period. For $\mathrm{BrO}^{-}$ions the results were obvious as first it underwent external diffusion followed by internal diffusion, thus higher initial slopes were observed in breakthrough curve for $\mathrm{BrO}_{3}^{-}$ions [14]. Whereas due to the absence of $\mathrm{Br}^{-}$ions in the initial stages there occurred a series of adsorption and reduction which helped in successfully removing the $\mathrm{Br}^{-}$ions. This shows that weaker physical force of attraction such as electrostatic force may be required to remove $\mathrm{Br}^{-}$ions which can be enhanced by ionic compression.

In the full scale column study it was observed that the breakthrough curve preceded that of RSSCT. The possible reasoning for this may be interferences by bio film activity. The best $\mathrm{BrO}^{-}$ions removal occurred at $\mathrm{pH}$ 6. For DOC the breakthrough preceded $\mathrm{BrO}^{-}$ions at $\mathrm{pH} 7$ and breakthrough curve was achieved in 1.4 days as compared to full scale breakthrough achieved in 42 days run. Mass balance of $\mathrm{BrO}^{-}$and $\mathrm{Br}^{-}$ions were determined at $\mathrm{pH} 7$ and bed volume of 6000 [14]. Spent GAC was washed in organic free water, $0.1 \mathrm{M} \mathrm{H}_{2} \mathrm{SO}_{4}$ and in $0.1 \mathrm{M} \mathrm{NaOH}$. The RSSCT result accounted for $70 \%$ and $59 \%$ removal of $\mathrm{BrO}_{3}^{-}$and $\mathrm{Br}^{-}$ions [14]. It was observed that the removal of $\mathrm{Br}^{-}$ions was higher as compared to $\mathrm{BrO}_{3}{ }^{-}$ions in $\mathrm{RSSCT}$ and the main reason of interference was found to the biological activity [14].

\subsection{Removal of Perchlorate by GAC using RSSCT}

Water supplies are highly contaminated with the presence of perchlorate in many part of the world which is as high as $37 \mathrm{ppb}$ [24]. The recommended concentration of perchlorate by EPA is $18 \mathrm{ppb}$ [24]. RSSCT has been used to predict the adsorption capacity of GAC in removal of per chlorates by ensuring that the experimental conditions such as flow rate, EBCT and carbon mass is well-matched with the full scale column study. The preloaded experiments of RSSCT with iron-organic complexing solution showed increased adsorption by GAC in removal of perchlorate. The efficiency of GAC increased by $20-25 \%$ when compared with the non pre loaded conditions [17]. The preloaded GAC was initially fried at a temperature of $105^{\square} \mathrm{C}$, which again increased the efficiency of GAC to 40-45\% [7].

RSSCT study was carried out to find the removal of trichloroethylene and dibromochloropropane from groundwater. The EBCT was calculated as 40 minutes, 0.49-0.59 bed volume and flow rate of 4 MGD [24]. The breakthrough was achieved in 6 months in RSSCT as compared to 18 months in pilot scale column. The groundwater before treatment contained about 68-138 ppb of Perchlorate [24]. The RSSCT columns gave good simulations of pilot scale column, which indicates high intraparticle diffusion. Proportional RSSCT column adopted for the experiment as it was found to be more representative form of full scale column and showed improved adsorption characteristics by GAC. The commercially available GAC showed less adsorptive capacity. The breakthrough of perchlorate started at a bed volume of 1200-1800 and full breakthrough was achieved at a bed volume of 2000-25000 with a EBCT of 20 minutes when bituminous GAC was used. But when preloaded GAC was used it showed improved adsorption as compared to the non preloaded one. For non preloaded GAC $80 \%$ breakthrough was achieved for the bed volume of 1780-1790 [24]. But for preloaded GAC $80 \%$ breakthrough was achieved for the bed volume of 2070-2220, showing enhancement of 16-20\% [24]. Exhausted GAC was regenerated by washing with a reducing solution. The desorbed wastewater contained perchlorate as high as 7000-15000 ppb and showed initial adsorption capacity of 50-74\% [24].

\subsection{Removal of Trace organic contaminant by GAC using RSSCT}

The most effective method in the removal of dissolved organic carbon (DOC) from drinking water is using GAC. Assessing pilot-scale GAC performance is dependent on time and high-priced. On the other hand adsorption is a difficult process to scale down which is limited by non steady state, and mass transfer [16]. RSSCT is a primary tool for evaluating GAC and for the removal of DOC in the presence of dissolved organic matter (DOM). The technology of RSSCT is highly dependent on EBCT and the type of GAC being used [17].

Adsorbents such as Bisphenol A and erythromycin were selected for the RSSCT experiment. Their molecular weights and $\mathrm{pKa}$ values were of 228 and $734 \mathrm{Da}$ and of 10.5 and 8.88 respectively [16]. The adsorbates were radiolabelled with 14C. Fresh bituminous GACs were used as adsorbents, which were crushed 
to obtain $d_{p}$ of $0.61 \mathrm{~mm}$ and after crushing it was washed, dried, and stored in a desiccators until used. The RSSCT method resulted in effluent with a concentration of $5 \mathrm{ng} / \mathrm{L}$ for bisphenol A and 15ng/L for erythromycin [16]. The samples from full scale column were analyzed using Pilot samples were analyzed using liquid chromatography followed by tandem mass spectrometry which showed the concentration of the effluent to be around $1 \mathrm{ng} / \mathrm{L}$. The DOC samples were analyzed in TOC analyzer using the UV irradiation at a wavelength of $253.7 \mathrm{~nm}$ for the equilibrium tests the spiking of Water samples were done with $500 \mathrm{ng} / \mathrm{L}$ Bisphenol [16]. This was then equilibrated for a period of 118 days for particle size of the $0.61 \mathrm{~mm} \mathrm{[16].} \mathrm{It} \mathrm{was} \mathrm{assumed} \mathrm{that} \mathrm{the}$ equilibration times should be proportional to the $\mathrm{d}_{\mathrm{p}}$ of GAC [16].

By using the approach of proportional diffusivities the RSSCT column was designed it helps in better prediction of breakthrough curves. The column of $4.76 \mathrm{~mm}$ internal diameter was packed with GAC with $\mathrm{d}_{\mathrm{p}}$ of $0.11 \mathrm{~mm}$ and $0.20 \mathrm{~mm}$ respectively and column of $7.0 \mathrm{~mm}$ was packed with $\mathrm{GAC}$ with $\mathrm{d}_{\mathrm{p}}$ of $0.61 \mathrm{~mm}$ [16]. The calibrated flow rate was found to be $2 \mathrm{~mL} / \mathrm{min}$. samples were collected every $24-48 \mathrm{~h}$ and in order to determine the throughput of sampling the water volume was measured in discharge tank was measured. The DOC concentration was analyzed in UV [16].

It was found that when the proportional diffusivities approach is used, it is not dependent on the $d_{p}$, as there was only a single breakthrough curve and equal surface loadings for all the three types of fractions used namely, $0.11 \mathrm{~mm}, 0.21 \mathrm{~mm}$ and $0.61 \mathrm{~mm}$ also it was found that the proportional diffusivities approach is useful stimulating the removal of DOM [16]. A continuous breakthrough curve shows that repacking of GAC has no effect on the mass transfer zone. Adsorption isotherm studies showed that there exists lower adsorption capacity when larger GAC particles are used which confirms direct site competition and pore blockage. But with smaller particle size the pore blockage is reduced because of increased surface area and bulk flow which in turn reduces the fouling of media. Because of all these factors the overall mass transfer may get reduced which results in the low adsorption capacity. Thus it was hypothesized that when the whole process is dependent on particle size then PD-RSSCT approach best in predicting the performance of the media and breakthrough curve [17].

\subsection{Capacity of GAC Preloaded with Natural Water}

Extensive studies have shown that a broad range of organic contaminants can be removed by using GAC. But sometimes the presence of background organic contaminant can reduce the performance of GAC by competing with the target compound. It has been found that when GAC is preloaded with natural water the adsorption capacity reduces drastically, which affects the scale-up techniques [18].

A study was done by spiking cis-1,2-dichloroethene (DCE) in source water which was then injected in column of pilot scale preloaded with un spiked water sample and effluent was collected at regular interval to determine the breakthrough. RSSCT were designed proportional to the columns of pilot scale column based on scaling principle developed by Crittenden et al 1986 [1]. The EBCT was designed for 20 minutes, the flow rate was maintained at $10 \mathrm{~m} /$ hour, the internal diameter of the column was $3.8 \mathrm{~cm}$ and the length of the column was $0.5 \mathrm{~m}$. after preloading the column were spiked with $400 \mathrm{ppb}$ of DCE [18]. The scaling relationship helped in scaling down the pilot scale study of 16 weeks to 4 weeks in RSSCT. During the experiments few variations were made to make the study more effect. The first method was to crush the wet media then the samples were decanted off and the carbon was filtered out on tarred $0.45 \mu$ which was then dried and weighed to find out the weight of the media used [18]. The second method was used to find that whether the capacity of media is improved by the removal of fouling matter. For this the preloaded carbon and water were shaking in a beaker for a particular time, and then the carbon was separated from water. It was found that there was no significant difference in the weight of the adsorbed natural organic matter and to the weight of the carbon. After the completion of experiment and obtaining the breakthrough, the analysis was done using a scanning electron microscope (SEM) [18].

The result showed similarity in the breakthrough profiles of RSSCT and pilot scale columns. But there was a significant difference in the breakthrough curve of a virgin-carbon column which was not preloaded with the source water. It was also found that the breakthrough curve for RSSCTs were longer than the pilot scale columns and the possible reason for it may be because of the assumptions made that surface diffusion coefficients are equal for both RSSCTs and pilot scale columns and also that the surface diffusion phenomena is dependent largely on the size of particles [17]. Those columns in which GAC preloaded with natural water has been used have breakthrough profiles dependent on and film transfer rate which changes at the top of the bed. A that medium which comes in immediate contact with the contaminant have reduced film transfer rate as the contaminant may get saturate the top of the bed reducing the rate of film transfer. Thus based on the knowledge of reduction in capacity and surface diffusion, accurate prediction can be made [17].

\subsection{Adsorption of RDX and HMX using RSSCT}

RDX (Royal Demolition Explosive or hexahydro-1,3,5-trinitro-1,3,5-triazocine) and HMX (High Melting Explosive or octahydro-1,3,5,7-tetranitro-1,3,5,7-tetrazocine) are explosives being used in armed forces 
and have been found as soil and groundwater contaminants. These chemicals are highly toxic to human health if present in the concentration of 0.002 and $0.4 \mathrm{mg} / \mathrm{L}$ for RDX and HMX, respectively as mentioned by the USEPA (2000). The most effective method in the removal of these toxic chemical compounds is the use of GAC which has been proved by adsorption equilibrium and kinetic studies. It is found that there is competition among these chemicals in aqueous solutions adsorption sites. The use of continuous flow column tests (RSSCT) confirms that the use of GAC can effectively remove the high explosive (HE) contaminant from the groundwater. At small laboratory scale RSSCTs can be performed for stimulating the performance of pilot scale column. RSSCTs were used to determine the performance of commonly used GACs and to determine the effect of preloading GAC with NOM present in groundwater. Based on USEPA (1994) quantification was done using high performance liquid chromatography (HPLC). $45 \%$ water and 55\% methanol was used as mobile phase under isocratic condition with a flow rate of $0.6 \mathrm{~mL} / \mathrm{min}$ [19]. C18 reversed phase column with a guard column was used with particle size of $5 \mu \mathrm{m}$, column length of $250 \mathrm{~mm}$ and internal diameter of $34.6 \mathrm{~mm}$. The detected peaks were quantified by measuring the absorbance at $254 \mathrm{~nm}$ in UV spectrophotometer. At about $6.7 \mathrm{~min}$ HMX was eluted and RDX eluted at about 10.2 min [19]. Total organic carbon (TOC) was measured using TOC analyzer. It was observed that the concentrations of groundwater NOM was $0.63 \mathrm{mg} / \mathrm{L}$ as TOC which was low, thus the surface diffusivities were assumed to be constant with respect to GAC particle size for RDX and HMX [19].

The RSSCT column was setup using chromatographic column having inner diameter of $30.8 \mathrm{~cm}$ and column length of $25 \mathrm{~cm}$ with a bed length of $5 \mathrm{~cm}$ for $1 \mathrm{~g}$ GAC [19]. Analysis of effluent samples was carried out for explosives and TOC to develop breakthrough curves. Groundwater was used as influent which was spiked with RDX $(2,200 \mu \mathrm{g} / \mathrm{L})$ and HMX $(350 \mu \mathrm{g} / \mathrm{L})$. RSSCT was used to study the effect of NOM on adsorption of RDX and HMX. For this $1.09 \mathrm{~g}$ of GAC, flow rate of $11.4 \mathrm{~mL} / \mathrm{min}$ and EBCT of $8.2 \mathrm{~min}$ was used. It was observed that in the uncontaminated groundwater the initial TOC concentration was $0.63 \mathrm{mg} / \mathrm{L}$ [19]. The average preloading of TOC onto the GAC was calculated by measuring effluent TOC concentrations. The groundwater was spiked with $2,200 \mu \mathrm{g} / \mathrm{L}$ of RDX and $300 \mu \mathrm{g} / \mathrm{L}$ of HMX after preloading, and then the breakthrough curves for both contaminants were determined by analyzing the effluent samples collected [19].

Adsorption isotherm studies were carried out using bottle-point isotherm tests, in a pre cleaned amber glass bottles of 250 - or $500 \mathrm{~mL}$, to obtain equilibrium liquid phase concentrations. After 1 week the samples were filtered and analyzed. Mass balance was used to determine the amount of compound to be loaded on GAC. The adsorption characteristics were of RDX and HMX was explained by Freundlich isotherm. Based on the assumptions of constant diffusivity the pilot scale column was scaled down to small scale column with flow rate of 23 to $43 \mathrm{~mL} / \mathrm{min}$ [19]. The predicted breakthrough time was about 3 days but the performance of RSSCT model was much better than the predicted results with average higher loading rate. The average hydraulic loading rate for RDX was $96 \mathrm{mg} / \mathrm{g}$ GAC [22]. The effluent HMX concentrations were not same as the influent HMX concentration which indicates additional adsorption capacity of GAC for HMX [19].

\subsection{Performance and Cost Analysis of RSSCT Technology}

RSSCT technology uses synthetic, polymeric and carbonaceous adsorbents in water treatment. It has been found to be effective in the removal of organic contaminants from the source water [20]. But the major problem is the high operating and maintaining cost of this technology. Recent advancements such as regeneration capacity through air stripping, solvent extraction, resistance to fouling and oven regeneration are making them very popular and demanding in the market [21].

These adsorbents used in RSSCT have a hydrophobic nature which gives them better adsorbent capacity. The performance of such system has increased three folds, with integrated column capacities. Organic adsorbents have relatively high adsorption capacity with greater affinity. As a result the effluent concentration exceeds that of influent concentration [20]. Even after the column gets saturated the level of effluent concentration tends to increase when compared to influent concentration level. Thus the performance of such technology is relatively high as compared to the other technologies which are used in treating water and in the removal of organic compounds [8].

\subsubsection{Cost Analysis of RSSCT Technology}

Cost analysis can be done by studying the concentration of contaminant getting removed, EBCT of the RSSCT technique used, influent and effluent concentration after treatment and the breakthrough curves. It has been found that a $50 \%$ increase in single breakthrough times has significantly increased the throughput of that experiment to a major extent [22]. The number of changes in technology per year is being used to study the change in operating and maintenance cost of this technology, which includes GAC replacement cost, cost of labour, transport and power used. 
The cost analysis has also helped in evaluating the post regeneration options, degradation of microbial action, steam stripping and disposal of hazardous waste. The breakthrough curves and the number of bed volume treated are used to predict the adsorption capacity and kinetics [23]. Higher treatment cost greatly depends on the higher NOM concentration when analysed for TOC concentration, which on turn is responsible for greater fouling and reduced performance [23]. But with the recent advancements such as regeneration capacity through air stripping, solvent extraction, resistance to fouling and oven regeneration are making them very popular and demanding in the market along with improved contaminant removal from the source water [23].

The main benefit is improved quality of water after treatment. Thus for cost effectiveness the media should be a passive one and highly selective [22]. Semi empirical approach should be adopted for cost effectiveness. The best way of managing the cost is by correlating the breakthrough between RSSCTs and pilot scale study being undertaken for the removal of particular contaminant and by determining the surface diffusion coefficient for the media being used to obtain better scaling relationships [23].

\section{Conclusion}

RSSCT is the most ideal method to predict the pilot scale column behaviour on a laboratory scale. This method of predicting the adsorption capacity of the media in the removal of contaminant requires the media to be grounded to desired size to obtain high efficiency. It has been found out from the previous studies stated above that there is no significant difference in surface morphology for the different sizes of media being used.

It has been used to evaluate the efficiency of adsorbents in removing contaminants from drinking water. It has been successfully used in contaminant removal such as in removal of arsenic using adsorbent media such as GAC, metal oxyhydroxides, activated alumina and ferric hydroxide. It has been also used in removal of bromated ions, perchlorate ions, trace organic contaminant, RDX and HMX by GAC. The efficiency of GAC preloaded with natural water has also been study to study the effect of any background organic contaminant on the adsorption capacity. RSSCTs facilitate the selection and optimization of media which directly affects the quality of water being treated. Both adsorption kinetics and adsorption capacities influences the performance of the media It has also facilitated the evaluation of packed bed operational modes in terms of parallel and series column arrangement in RSSCT. It has been found that pilot scale column works intermittently and the breakthrough achieved in RSSCTs is expected to be similar to that of the pilot scale studies.

It has been found that the media which is used in removal of arsenic has also the capacity of removing phosphorous and vanadium and the iron based media has the best performance in arsenic removal. The most valid approach for the study of adsorption by porous media is PD approach. It shows rapid adsorption for media with small particle size. The above studies shows that the adsorption capacity of RSSCTs extends beyond adsorption of trace organic contaminant as now a days it is used in removal of inorganic contaminants such as arsenic, uranium, vanadium, perchlorates on porous metal adsorbents apart from using GAC as the sole adsorbers.

The removal of bromated ion by GAC in RSSCT showed that the breakthrough of DOC RSSCT preceded that of full scale column test and also the breakthrough of bromated ions and the possible reason thought to be responsible for this was the interference by the activity of bio films formed on the surface of the media. RSSCT gives a good prediction model for the removal of bromated ions and can be successfully used to stimulate the pilot scale experiments. It is also found that adsorption of bromide is weaker because of the electrostatic force of attraction. The performance of GAC in the removal of RDX and HMX has also been successfully acknowledged.

It has been found that preloading GAC with natural water or contaminated water reduces the rate of film transfer on the top of the bed in both RSSCTs and pilot scale columns. But those columns which are fed with the contaminated water at start don't show any significant difference in film transfer rate as it may become saturated with the contaminant.

The review on previous works gives a significant comparison of RSSCTs and pilot scale column experiments for obtaining the breakthrough profiles of DOC. The review work can be concluded by saying that there could be some interesting advancements in the treatment of drinking water with this adsorptive technologies which are being developed now a days. Development in the mechanism for removal of uranium can lead to the development of advanced ion exchange resins. Development of adsorptive media based on nanotechnology can lead to revolutionary outcomes in this field with increased treatment of contaminants. Development of nano engineering adsorptive media can lead to removal of target specific contaminants thus obtaining high efficiency and performance. The relationship between surface diffusivities and size of particle will help in reducing the impact of fouling by NOM present in groundwater and will increase the adsorption effiency of high explosives such as RDX and HMX which have been recently identified as the source of groundwater pollution in armed force and military areas. 


\section{Acknowledgements}

The authors would like to sincerely thank Environmental, Water Resources \& Transportation Engg. Division, SMBS, VIT University, faculty of SMBS, Chancellor of VIT University for their brilliant innovative ideas and support.

\section{Journal Papers:}

\section{References}

[1] J. C. Crittenden, J. K. Berrigan, and D. W. Hand, Design of rapid small-scale adsorption tests for a constant diffusivity, J. Water Pollution. Control Fed, 58(4), 1986, 312-319.

[2] Crittenden J. C. Crittenden, J. K. Berrigan, and D. W. Hand, Design of rapid fixed-bed adsorption tests for non constant diffusivities, J. Environ. Eng, 113(2), 1987, 243-259.

[3] L. Yu, C. Adams, and D. Ludlow, Adsorption Isotherms for Methyl Tert-Butyl Ether and Other Fuel Oxygenates on Two Bituminous - Coal Activated Carbons, J. Environ. Eng, 131(6), 2005, 0733-9372.

[4] L. Cummings, and R. S. Summers, To predict field-scale GAC control of DBP formation using RSSCT, J. Am. Water Works Assoc, 86(6), 1994, 88-97.

[5] P. Westerhoff, D. Highfield, M. Badruzzaman, and Y. Yoon, Rapid Small-Scale Column Tests for Arsenate Removal in Iron Oxide Packed Bed Columns, J. Environ. Eng, 131(2), 2005, 0733-9372.

[6] R. Rodriguez-Fuentes, B. A. Hilts, and B. I. Dvorak, Disinfection by-product precursor adsorption as function of GAC properties: Case study, J. Environ. Eng, 131(10), 2005, 1462-1465.

[7] A. G. Patni, D. K. Ludlow, and C. D. Adams, Characteristics of Ground Granular Activated Carbon for Rapid Small-Scale Column Tests, J. Environ. Eng, 134(3), 2008, 0733-9372.

[8] D. W. Hand, J. A. Herlevich, D. L. Perram, and J. C. Crittenden, Synthetic adsorbent versus GAC for TCE removal, J. Am. Water Works Assoc, 86(15), 1994, 64-72.

[9] D. R. U. Knappe, V. L. Snoeyink, P. Roche, M. J. Prados, and M.M. Bourbigot, The effect of preloading on rapid small-scale column test predictions of atrazine removal by GAC adsorbers, Water Res, 31(11), 1997, 2899-2909.

[10] S. Kunzru, and M. Chaudhuri, Manganese Amended Activated Alumina for Adsorption/Oxidation of Arsenic, J. Environ. Eng., 131(9), 2005, 1350-1353.

[11] B.I. Dvorak, M. Morley, and P. Denning, Relative Impact on GAC Usage Rates of Operating Strategies for Treatment of Contaminated Groundwater, Practice Periodical of Hazardous, Toxic, and Radioactive Waste Management,12( 2), 2008, 60-69.

[12] L. Ronald, Jr. Vaughan, B.E. Reed, and E.H. Smith, Modelling As (V) Removal in Iron Oxide Impregnated Activated Carbon Columns, J. Environ. Eng, 133(1), 2007, 0733-9372.

[13] W.F. Chen, S.Y. Lin, and M.T. Cheng, Rapid small scale column tests on the adsorption of arsenate by cationic surfactant -modified GAC, J. Environ. Eng, 131(4), 2012, 1943-7870.

[14] T.F. Marhaba, Examining bromate ion removal by GAC through RSSCT and pilot-scale columns, Environ Engg and Policy, 2(2), $1998,59-64$.

[15] N. Ates, U. Yetis, and M. Kitis, Effects of Bromide Ion and Natural Organic Matter Fractions on the Formation and Speciation of Chlorination By-Products, J. Environ. Eng, 133(10), 2007, 0733-9372.

[16] C. J. Corwin, and R.S. Summers, Scaling Trace Organic Contaminant Adsorption Capacity by Granular Activated Carbon, Environ. Sci. Technol, 44(14), 2010, 5403-5408.

[17] A. Bradley, R. Zachman, and S. Summers, Modelling TOC Breakthrough in Granular Activated Carbon Adsorbers, J. Environ. Eng, 136(2), 2010, 0733-9372.

[18] T. F. Speth, Evaluating capacities of GAC preloaded with natural water, J. Environ. Eng, 117(1), 1990, 0733-9372.

[19] C. Matthew, M. Morley, J. L., Henke, E. Gerald and Speitel Jr, Adsorption of RDX and HMX in Rapid Small-Scale Column Tests: Implications for Full-Scale Adsorbers, J. Environ. Eng, 131(1), 2005, 0733-9372.

[20] C. Volk, L. Wood, B. Johnson, J. Robinson, H. W. Zhu and L. Kaplan, Monitoring dissolved organic carbon in surface and drinking waters, J. Environ. Monit, 4(1), 2002, 43-47.

[21] J. Sutherland, C. Adams, and J. Kekobad, Treatability of Alternative Fuel Oxygenates Using Advanced Oxidation, Air Stripping, and Carbon Adsorption, J. Environ. Eng, 131(4), 2005, 0733-9372.

[22] S. Suresh, V.C. Srivastava, and I.M. Mishra, Adsorption of Hydroquinone in Aqueous Solution by Granulated Activated Carbon, $J$. Environ. Eng., 137(12), 2011, 145-1157.

[23] T. Shih, M. Wangpaichitr and M. Suffet, Performance and Cost Evaluations of Synthetic Resin Technology for the Removal of Methyl Tert -Butyl Ether from Drinking Water, J. Environ. Eng, 131(3), 2005, 0733-9372.

\section{Proceedings Papers:}

[24] F.S. Cannon and C. Na, Perchlorate Removal using Tailored Granular Activated Carbon and Chemical Regeneration, Proceedings of the Conference - Perchlorate Workshop of the Pollution Prevention Technology Transfer- 2000 organized by The Joint Armed Services in San Antonio, 2000. 